\title{
Experimental Dynamic Identification Of Modeshape Driving Wind Turbine Grid Loss Event On Nacelle Testrig
}

\author{
J.Helsen \\ Vrije Universiteit Brussel Pleinlaan21050 Brussel Belgium jan.helsen@vub.ac.be \\ C.Devriendt \\ OWI Lab/VUB Pleinlaan 2 1050 Brussel Belgium Christof.Devriendt@owi-lab.be \\ W.Weijtjens \\ OWI Lab/VUB Pleinlaan21050 Brussel Belgium wout.weijtjens@vub.ac.be \\ P.Guillaume \\ Vrije Universiteit Brussel Pleinlaan 21050 Brussel Patrick.guillaume@vub.ac.be
}

\begin{abstract}
This paper experimentally investigates a grid loss event on the Gearbox Reliability Collaborative drivetrain mounted on the NREL nacelle testrig. It is shown that during the grid loss event the system vibration is driven by a counter phase rotation of the rotor and generator rotor about the drivetrain flexibility. This behavior results in significant torque oscillations with significant negative torque periods. This work shows that at each zero-crossing the teeth disengage contact, go through the backlash and re-engage at the other flank by means of strain gauge measurements at the HSS pinion teeth. The driving torsional resonance is identified by means of a pLSCF modal identification estimator. Challenges for accurate modal parameter estimation related to harmonic excitation are elaborated and tackled. Finally the dominating eigenfrequency, corresponding modeshape and damping value are determined.
\end{abstract}

Keywords:

Wind Turbine, Emergency Stop, Operational Modal Analysis, System Identification

Preprint submitted to Renewable Energy

February 19, 2015

C) 2015. This manuscript version is made available under the Elsevier user license http://www.elsevier.com/open-access/userlicense/1.0/ 


\section{Introduction}

The wind turbine industry is continuously improving turbine designs to reduce the overall cost of wind energy. At the same time turbine reliability needs to be guarded in order to guarantee a lifetime of 20 years. Expert insights in loading conditions are one of the main requirements for achieving this goal. The loads to which the turbine is subjected are fluctuating due to the stochastic nature of the wind [1] and cannot be assumed to be quasi-static as in most industrial applications[13]. Wind turbine loading includes aerodynamic loads at variable wind speeds, gravitational loads and corresponding bending moments, inertial loads due to acceleration, centrifugal and gyroscopic effects, operational loads such as generator torque and dynamic loads induced by certain control actions like blade pitching, starting up, emergency braking or yawing $[4,5]$. In addition, loads caused by turbulence can result in unbalanced aerodynamic loads on different sides of the rotor. These loads lead to non-torque bending moments that feed into the drive train and induce non-symmetrical loads within the gearbox. The nature and importance of these non-symmetrical loads is significantly determined by the drive train configuration $[3,6,7]$.

Field measurements and dynamometer testing are used to validate simulation models for dynamic load prediction $[8,9]$. Simulation models as well as testrig validation campaigns require the definition of appropriate loading conditions as input. For initial model validity analysis it is suggested to use quasi-static operating conditions. However, dynamic events such as start-ups, shut downs and emergency events can significantly reduce the overall drivetrain life and are therefore important to be investigated. For example rapid load variations combined with misaligned gear shafts in the high speed stage can lead to undesired axial load cycles at the bearings [10]. The main design load cases, including the dynamic ones, are defined in standard IEC 61400-1 [11]. In addition standard IEC 61400-4 WTs-Part 4: design requirements for WT gearboxes[12] includes requirements for normal gearbox operation. Examples are starting and stopping 
procedures at cut-in, rated and cut-out wind speed linked to the control system concept. Another example is the control strategy for emergency braking procedure after activation of the safety system under grid failure. The definition of the controller strategy has significant influence on the overall turbine behavior. An example of such an event is the grid loss event. It is initiated by the loss of the electricity grid, followed by an emergency stop of the turbine. One of the main drivers in this event is the main torsional drivetrain resonance. This paper identifies this driving resonance frequency on the NREL 2.5MW nacelle dynamometer for the Gearbox Reliability Collaborative (GRC) drivetrain[14]. To do the identification a severe grid loss type event is simulated. The emergency stop of the dynamometer is activated to disconnect the generator from the grid and bring the speed of the drivetrain to zero in a controlled manner. The drivetrain can be interpreted as a torsional spring between the rotor inertia and generator rotor inertia. During this event the torsional energy stored in the system is released by decompression of this spring.

\section{Study approach}

The main goal of this paper is the identification of the main torsional resonance frequency of the GRC drivetrain on the NREL nacelle dynamometer. The corresponding modeshape is characterized by the counter-phase rotation of the rotor and generator inertias connected by the drivetrain flexibility, as schematically shown in Figure 1.

In the case of the nacelle testrig no full size rotor is present, as is the case in a wind turbine. This lower hub inertia will significantly affect the frequency value corresponding to the first torsional modeshape. However, the main goal of this paper is to show typical behaviour during a grid loss event and more particularly show that it is driven by this drivetrain mode. The change in frequency due to the extra inertia therefore does not influence the core message of this work. Since testrig testing offers significantly more controllability, it is opted to use dynamometer results in this work. The use of a full nacelle test-rig makes that 
the relevant boundary conditions, other than the rotor inertia are similar as in the turbine, while keeping the convenience of test-rig testing.

The drivetrain resonance is excited by means of a severe grid loss excitation by disconnecting the generator instantaneously from the electricity grid after which the speed of the system is reduced to zero in a prespecified control mode. In contrast to the grid loss event on a field turbine, the speed reduction is realized slowly in order to be able to clearly identify the resonance. The eigenfrequency and corresponding modeshape are identified by means of a polyreference leastsquares complex frequency-domain (pLSCF) estimator [15]. Moreover, gear contact behavior during the dynamic event is investigated by means of strain gauges at the root of the High Speed Shaft teeth. The goal is to identify the first drivetrain resonance frequency, which drives the torque reversals during which the gear contact is lost and the teeth contact the opposing flank. Harmonic excitation poses significant challenges in the modal identifcation process. These challenges are identified and the first drivetrain resonance determined.

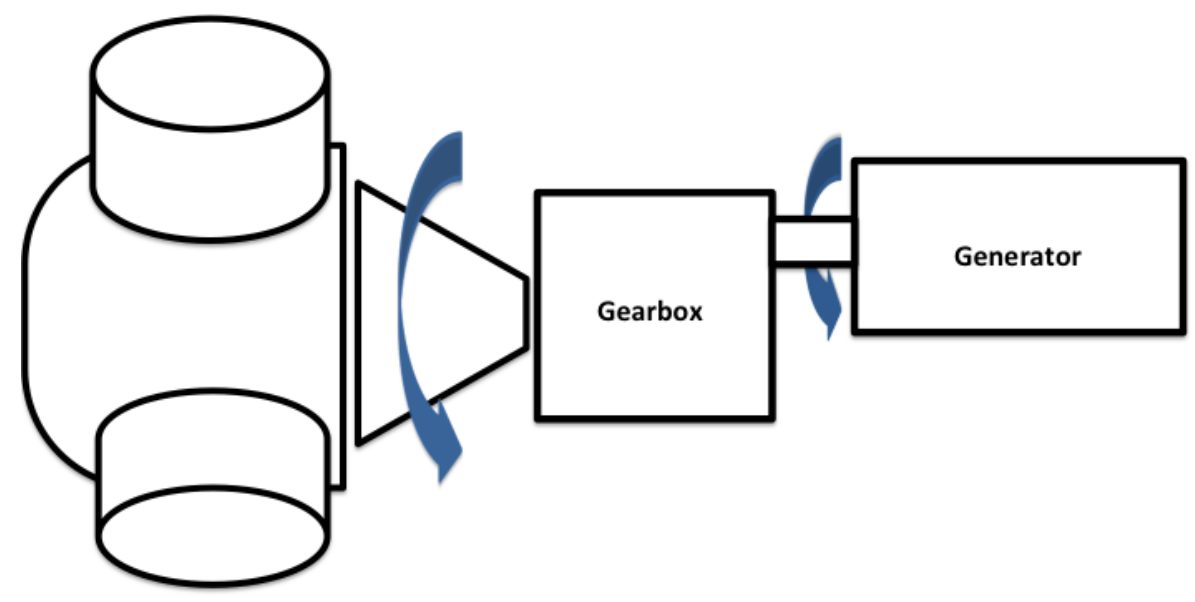

Figure 1: Schematic representation of modeshape corresponding to driving modeshape 


\section{Test object}

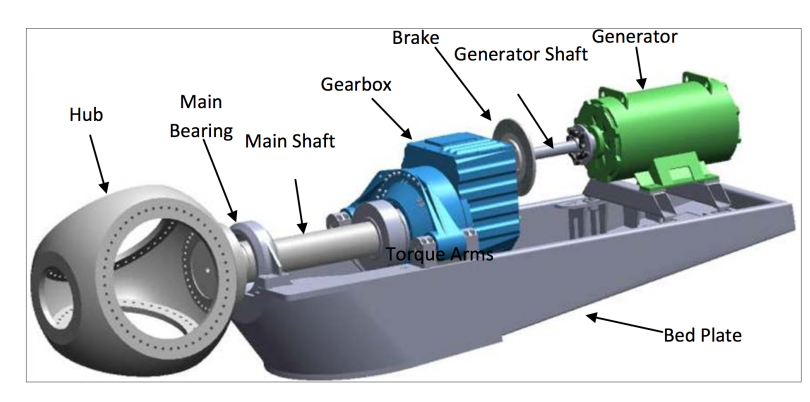

Figure 2: Nacelle of the GRC wind turbine [2]

\subsection{Test article description}

The drivetrain used in this investigation was designed in the framework of the GRC and intended to be installed in a stall-controlled, three-bladed upwind turbine with a rated power of $750 \mathrm{~kW}$. It has two rotor speeds, $14.7 \mathrm{rpm}$ and 22.1 rpm. The gearbox converts these speeds to generator speeds of 1,200 rpm and $1,800 \mathrm{rpm}$ achieving a ratio of 81.491 . The GRC turbine follows a conventional, three point mounting modular configuration. These nacelle components are the hub, main bearing, main shaft, gearbox, brake, generator shaft, and generator as shown in Figure 2. The GRC gearbox is designed based on current best practices and consists of one planetary gear stage and two helical gear stages. Figures 3 and 4 show the overall gearbox layout. Turbine and gearbox properties are listed in detail in Table 1.

The gearbox consists of one planetary and two helical gear stages. The planetary stage uses a cage planet carrier and a floating sun for optimal load sharing amongst the planets. The floating sun is connected to the first helical stage shaft by means of an articulated spline connection. 


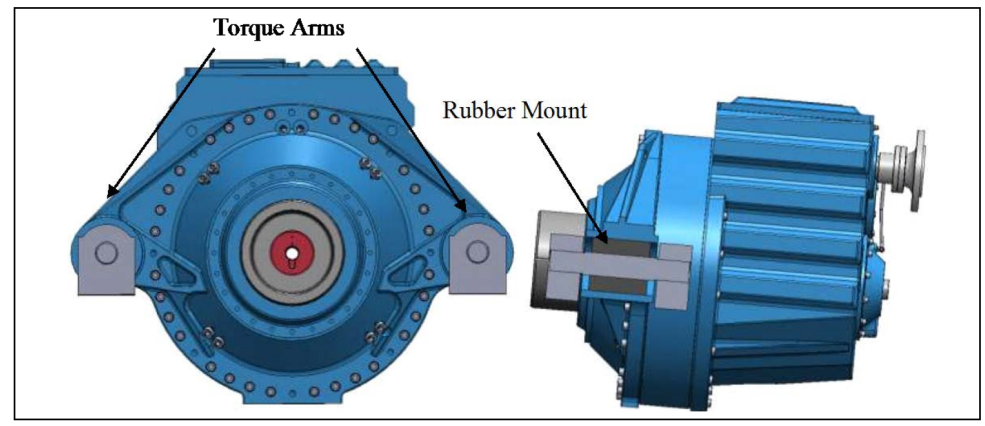

Figure 3: GRC gearbox [2]

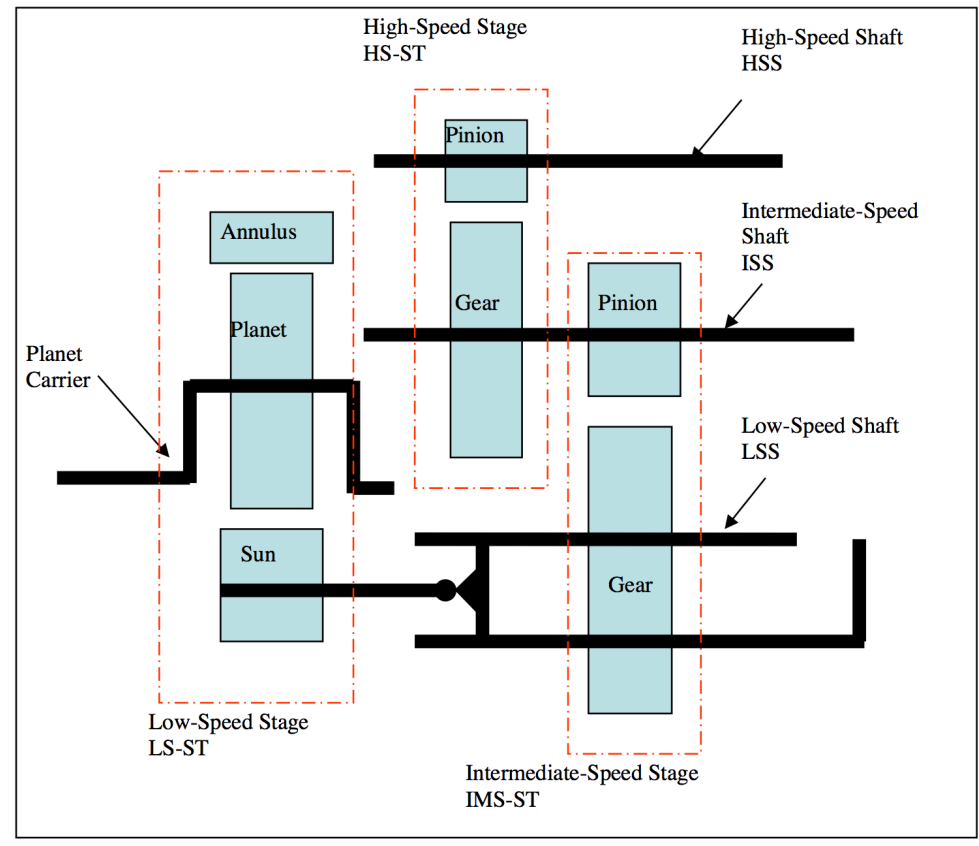

Figure 4: GRC gearbox topology[2] 
Table 1: GRC Drivetrain properties[2]

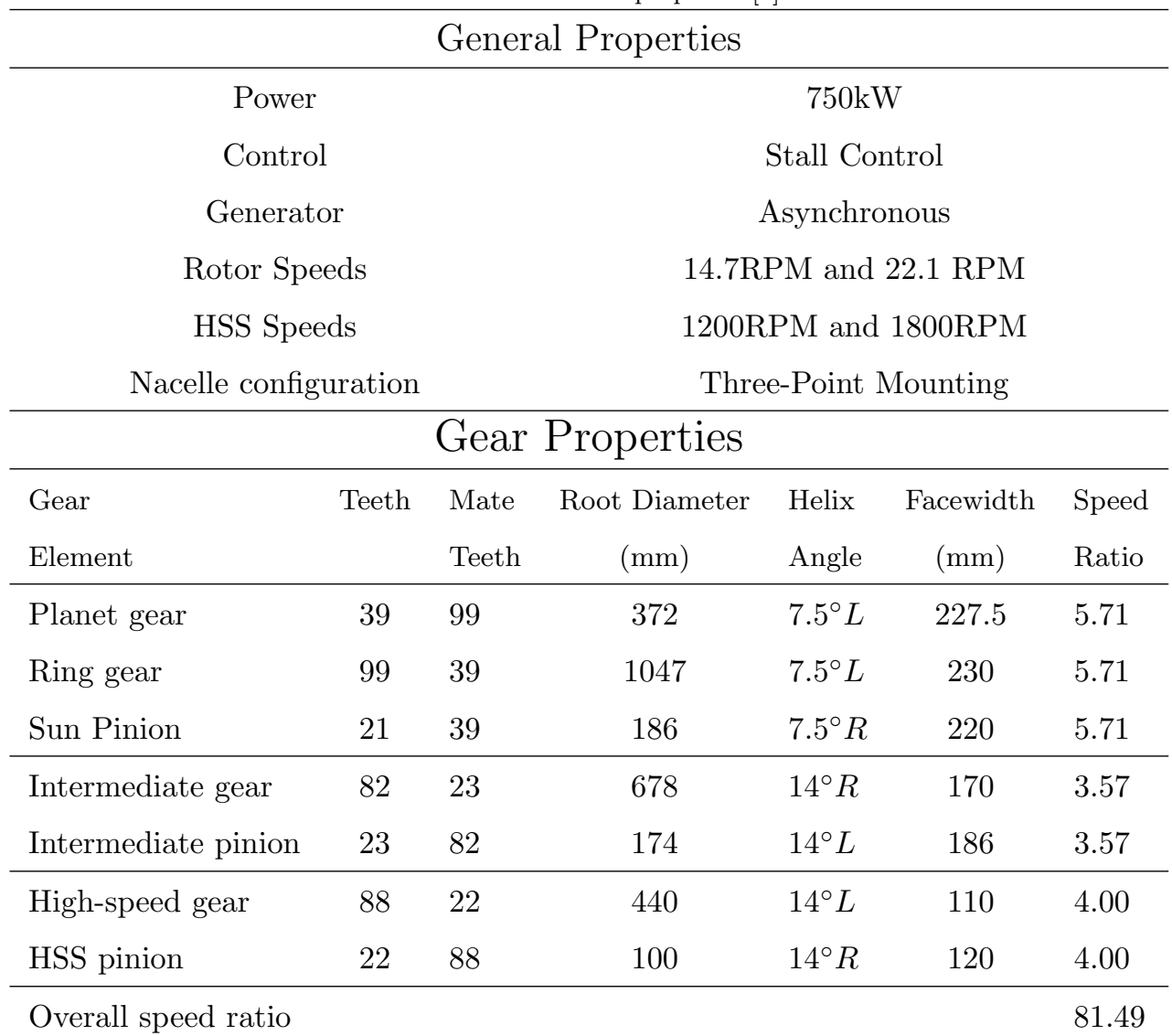




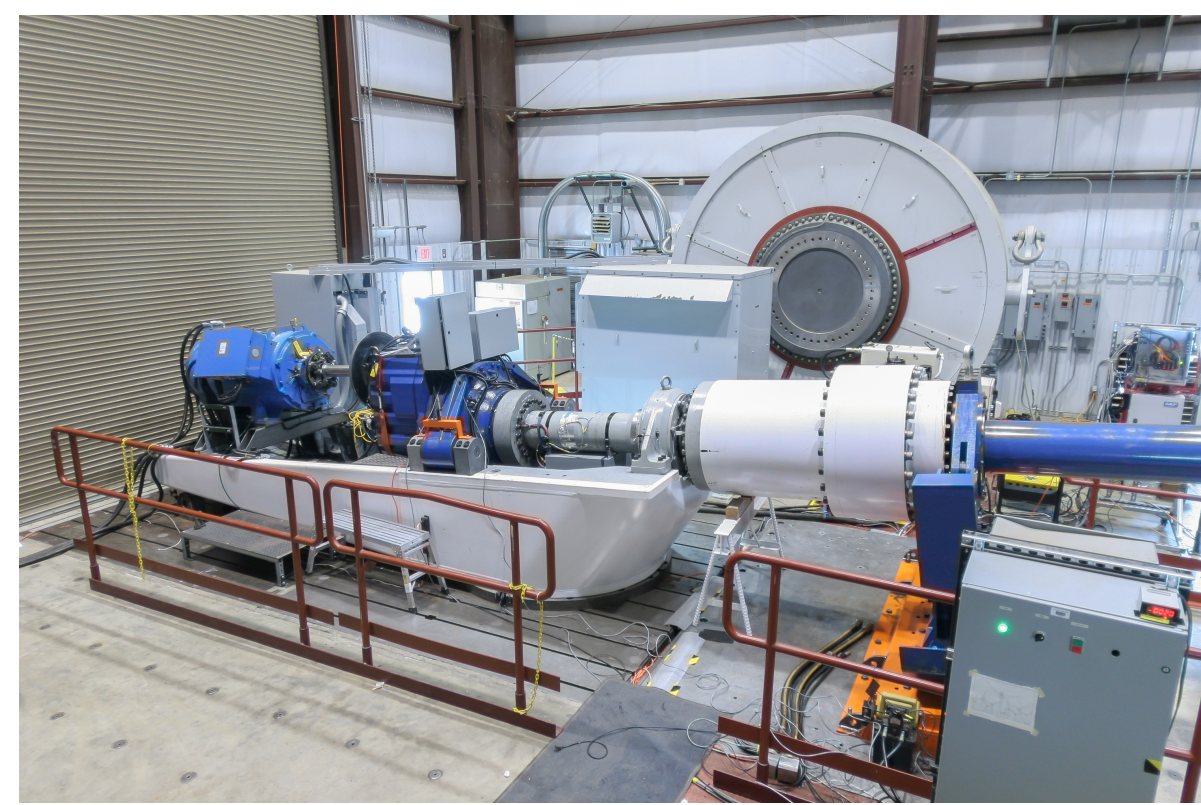

Figure 5: Experimental set-up on 2.5MW nacelle test-rig.Photo credits: NREL 24914, Lee Jay Fingersh

\subsection{Set-up and Instrumentation}

The complete nacelle is tested in the dynamometer, shown in Figure 5. It consists of a driving electric motor combined with gearbox to introduce mechanical power at the nacelle hub. This allows the GRC drivetrain to produce electricity which is fed back into the local grid, as schematically shown in Figure 6. Main advantage of this configuration is that the investigated nacelle controller stays active and the turbine produces energy as if it were in the field.

The electric micro-grid is managed by a control unit that allows the variation of both grid frequency and amplitude. 


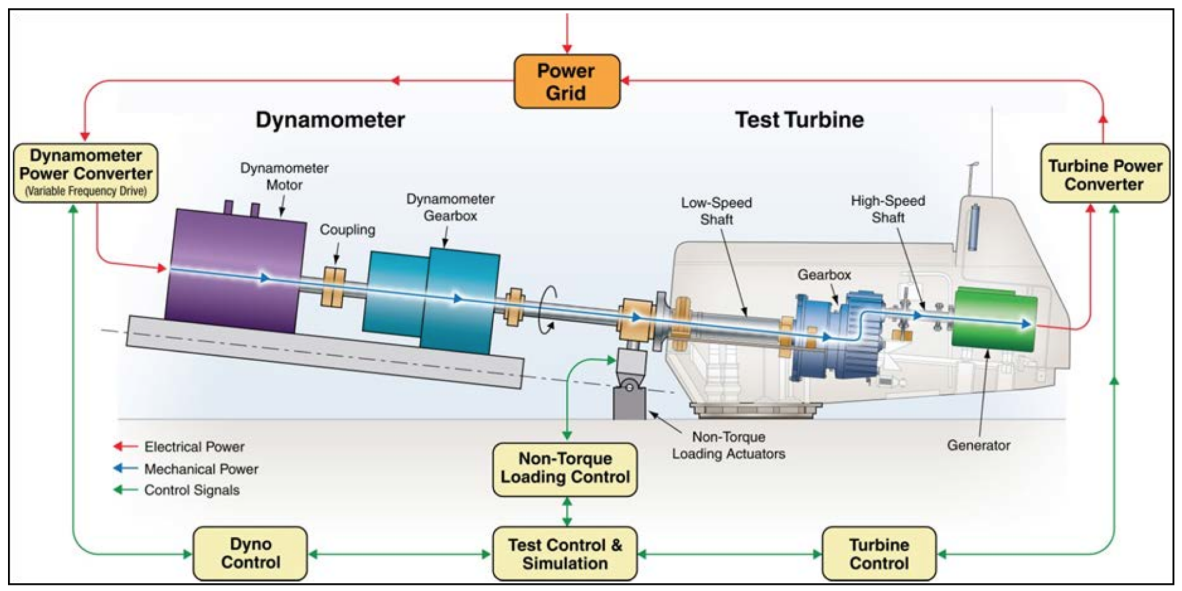

Figure 6: Schematic of the NREL 2.5MW nacelle test-rig [2]

characterize overall behavior two strain gauge bridges are used to characterize the torque fluctuations at the drivetrain main shaft (LSS), - the input of the gearbox-, and at the High Speed Shaft (HSS),--the output of the gearbox-'. Figure 7 shows the location of these bridge and their layout.

In addition to the torsional resonance behavior the tooth contact properties are of particular interest. More in particular the unloading of teeth during the dynamic event and the link to the applied torque loads are investigated. Strain gauge bridges in the root of the teeth of the HSS, shown in Figure 8 are used. Two bridges are used at a different center position. Since the HSS pinion teeth are helical the strain bridges are placed according to the helix angle. The advantage of using these two bridges is the ability to asses load distribution in the direction of the tooth width.

\section{Dynamic event description}

The load case used in this paper is a grid loss event. Initially the drivetrain operates at normal conditions. After 3.2s the dynamometer emergency stop is activated, resulting in a disconnection of the electricity grid. At that instance the generator induced counter torque is lost. The RPM is brought to zero 


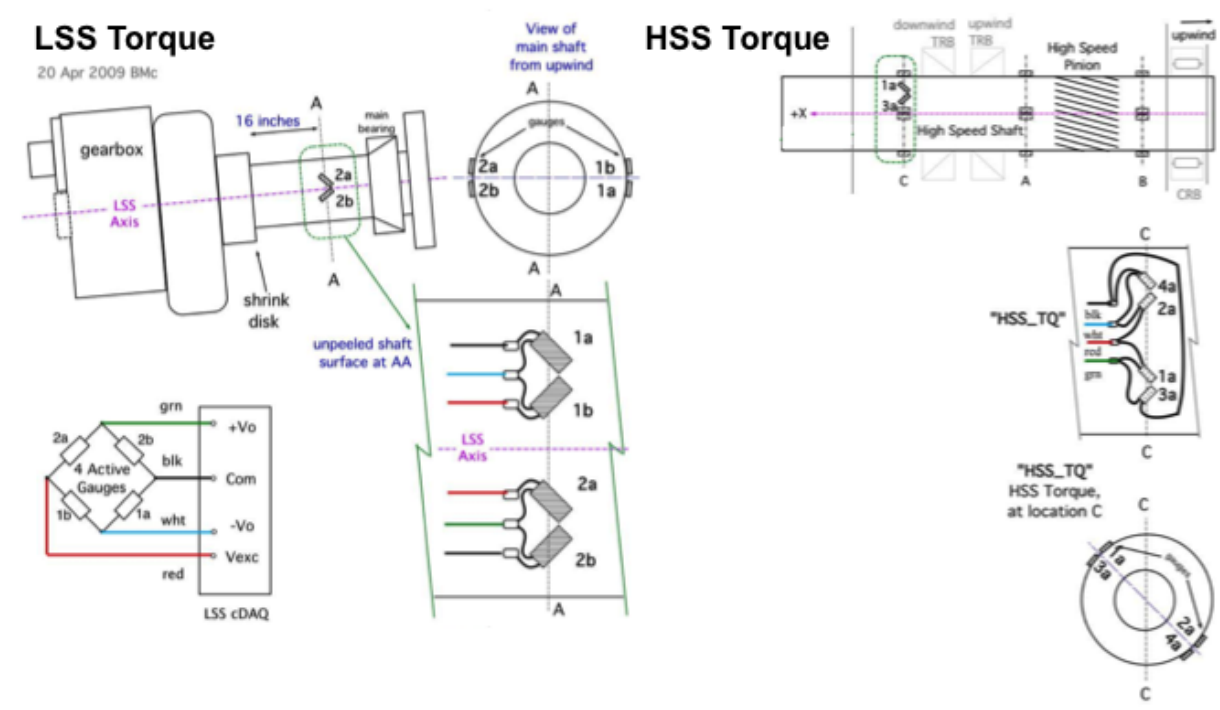

Figure 7: Low and High speed shaft strain gauge bridge for torque [2]

in a controlled slow manner over 3 minutes time. Figure 9 clearly shows the instantaneous decrease of active and reactive power to zero. Correspondingly the speed of the drivetrain starts to decrease in a controlled manner by the controller and a torque reversal is initiated. Figure 10 illustrates the torque and RPM behavior. RPM signals are measured at the HSS, whereas the torque signal originates from the testrig torque sensor. All quantities are normalized for easy comparison. The torque starts at $25 \%$ of the drivetrains nominal value, whereas the speed is at nominal RPM. During the first seconds of the transient the torque pulses between its initial normal value and a negative maximum equal to approximately $80 \%$ of this initial value. The torque fluctuations continue until full stop. Figure 11 shows the Fast Fourier Transform of the non-constant part of the torque signal starting at second 3.2 until second 18. There is clear excitation in the lower frequency range below $1 \mathrm{~Hz}$. The resonance frequencies of a full turbine in this range are predominantly related to tower motions and the first drivetrain eigenfrequency. Since no tower is present on the test-rig it is expected that the first drivetrain resonance frequency is driving the event. 


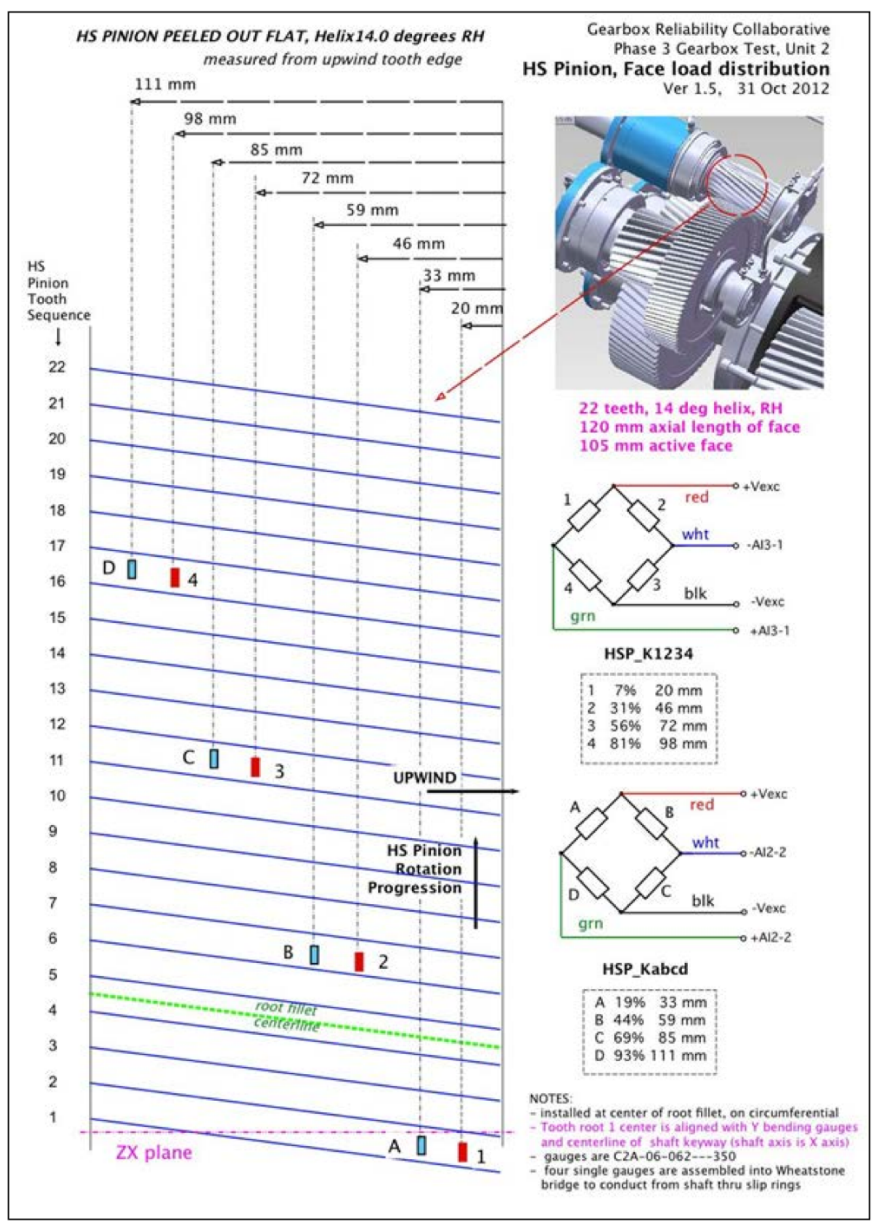

Figure 8: High speed shaft pinion face width tooth load distribution sensors: Bridge One: HSP-KABCD, Bridge Two: HSP-K1234 [2] 


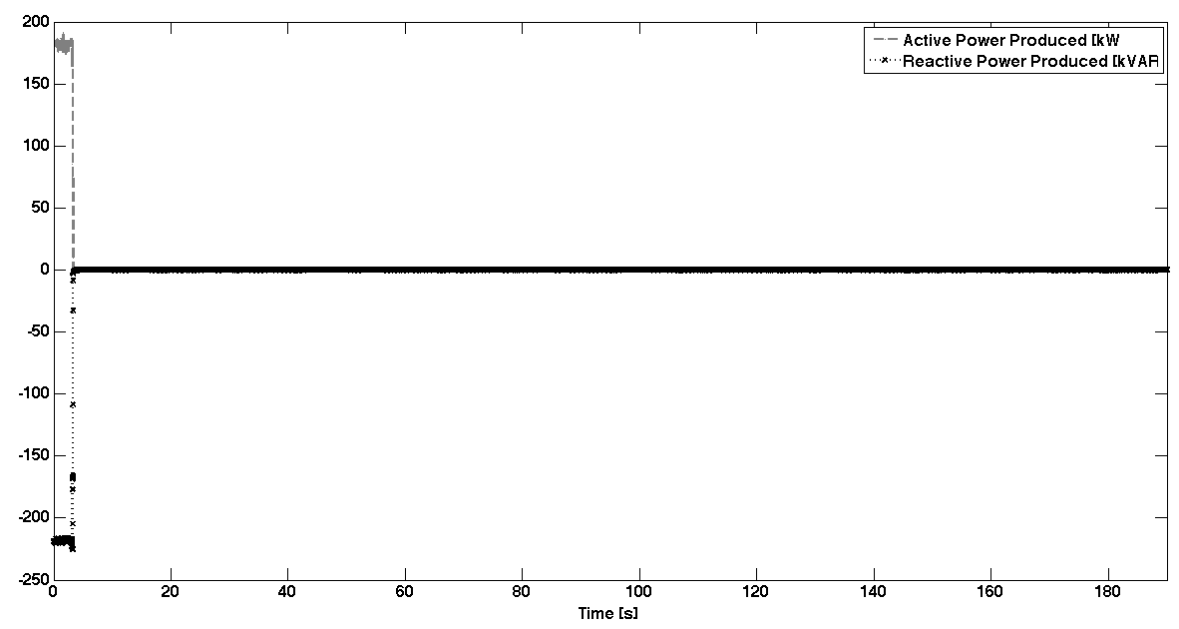

Figure 9: Active and reactive power produced during grid loss event

Advanced operational modal analysis will be used to identify this modeshape and its corresponding eigenfrequency and damping value.

\section{Gear loading}

In Figure 11 it can be seen that the torque is constant during a short period between the different torque reversals. Each of these constant parts corresponds to the gears going through their backlash. This is confirmed by the strain gauge measurements at the HSS teeth. Figure 12 shows the normalized strain of one of the two HSS tooth strain bridges plotted in combination with the normalized torque signal. The tooth strains show similar behavior as the torque signal. Zooming in on the initial dynamics illustrates this, as shown in Figure 13. A clear beating phenomenon is seen in the two HSS tooth strain bridge strains. The fast fluctuating strain signal is related to the meshing of the gears. Teeth engage into contact and disengage. Since the strain gauge bridge is divided over four teeth, as shown in Figure 8 four distinct peaks are present for each revolution of the gear. Figure 13 zooms in on the strain signals. The four distinct peaks related to each revolution are clearly visible. The beating phenomenon is a clearly visible in the envelope of the high frequency strain signal. The 


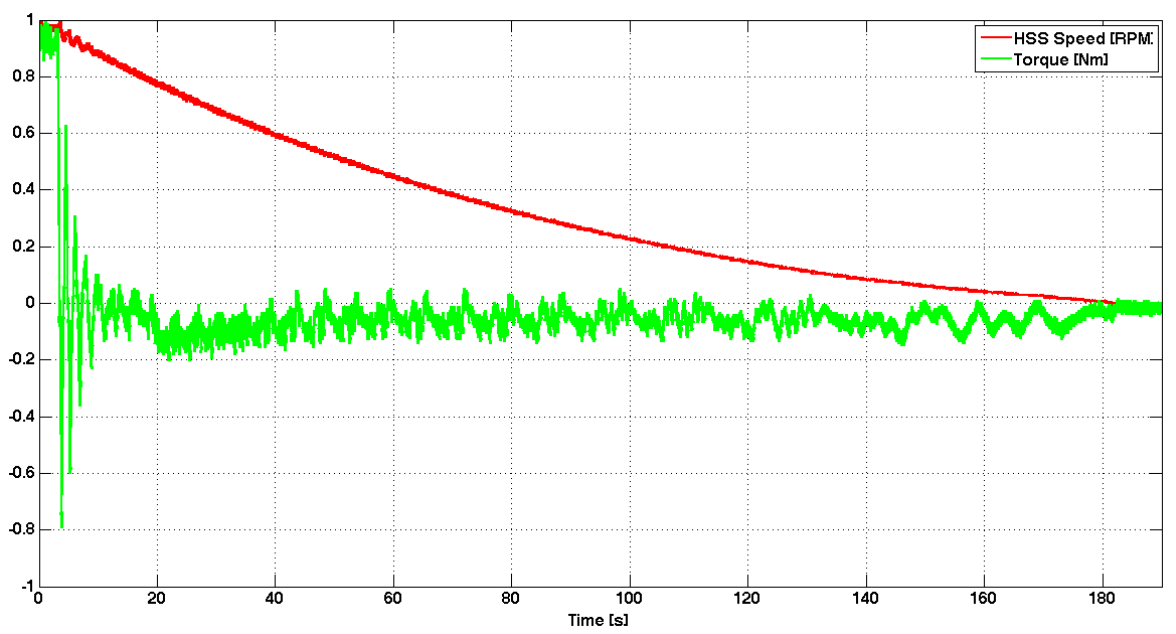

Figure 10: Normalized torque and RPM signal for grid loss event
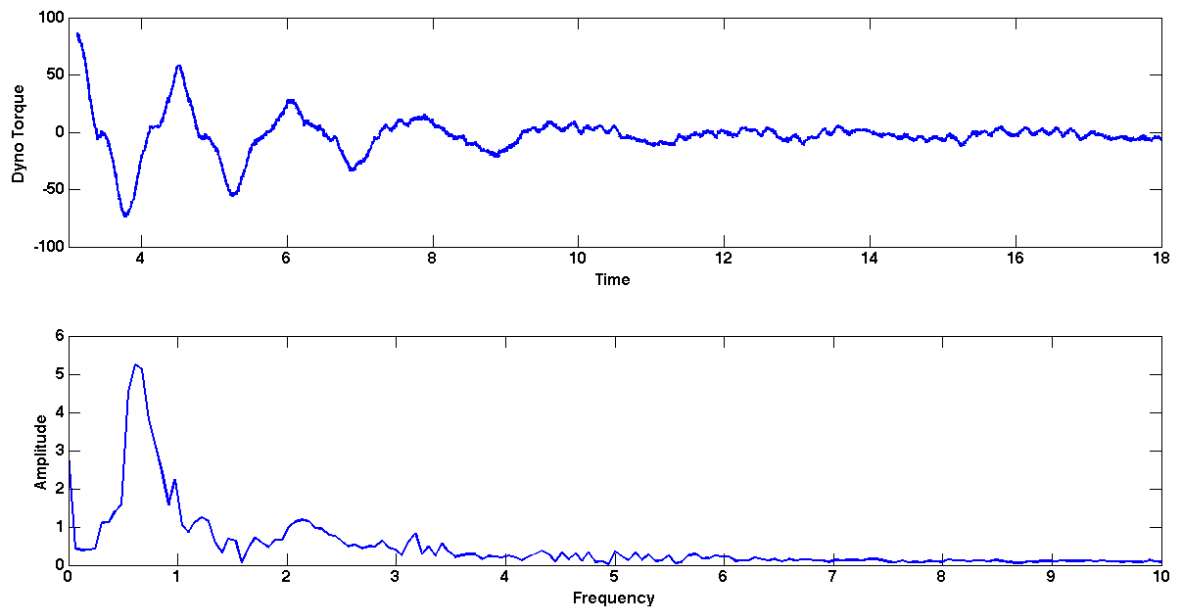

Figure 11: Torque oscillation and corresponding frequency excitation spectrum 
envelope is related to the overall fluctuation in the load on the teeth. By means of demodulation it is possible to extract the envelope signals from the original signals. These are plotted in Figure 14. The envelope signals that result from the demodulation process are clearly determined by the same trend as the torque reversals. Tooth loading is first decreasing towards zero, staying constant and increasing again to a maximum. Consequently it is decreasing again and so on. In between each of the beating events the teeth loose contact and are in backlash, as illustrated in Figure 14. Since at the same time the torque experienced by the test-rig changes sign the teeth are initiating contact at the non-driving flank while they are still at high speed. During the first seconds of the event the speed has only changed with $2.5-5 \%$. The negative counter torque generates a braking effect, whereas the positive torque results in a speed increase. The speed fluctuation is limited and shown in Figure 13. At each torque reversal the teeth are experiencing an impact loading. The amplitude of these impacts may not be present in the strain measurements, since not all teeth are instrumented and it is not known whether the instrumented teeth are the ones participating in the initial engagement. Based on the envelope signals it can be concluded that the torque fluctuation is mainly determining the event.

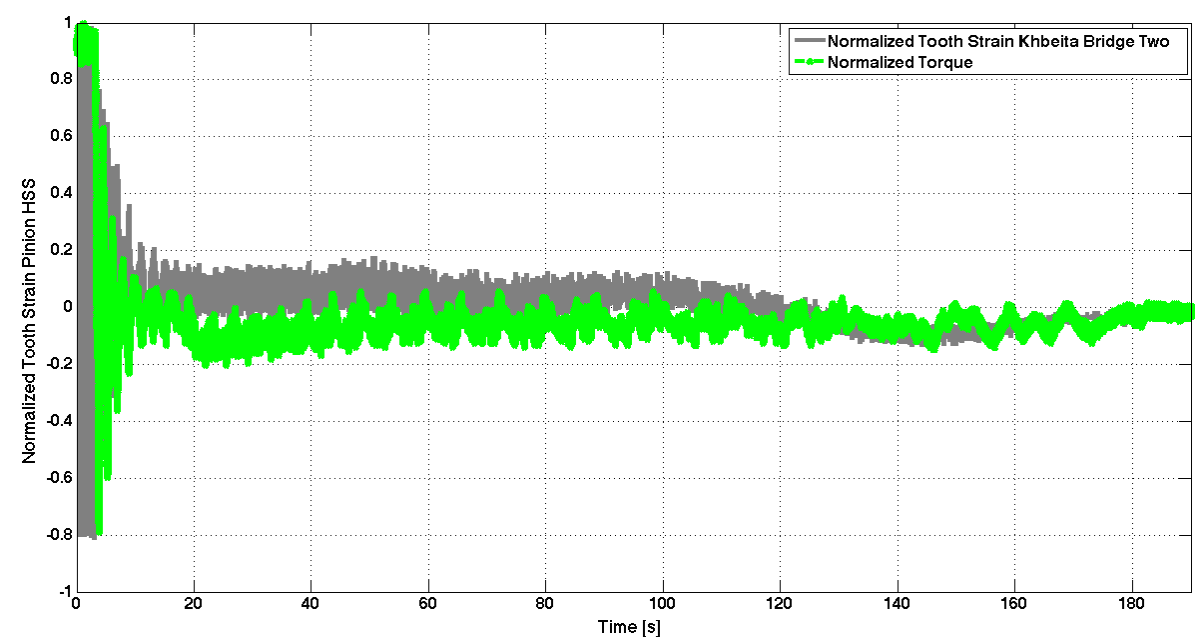

Figure 12: Tooth strains for gauge bridge 2 combined with normalized torque signal for grid loss event. 


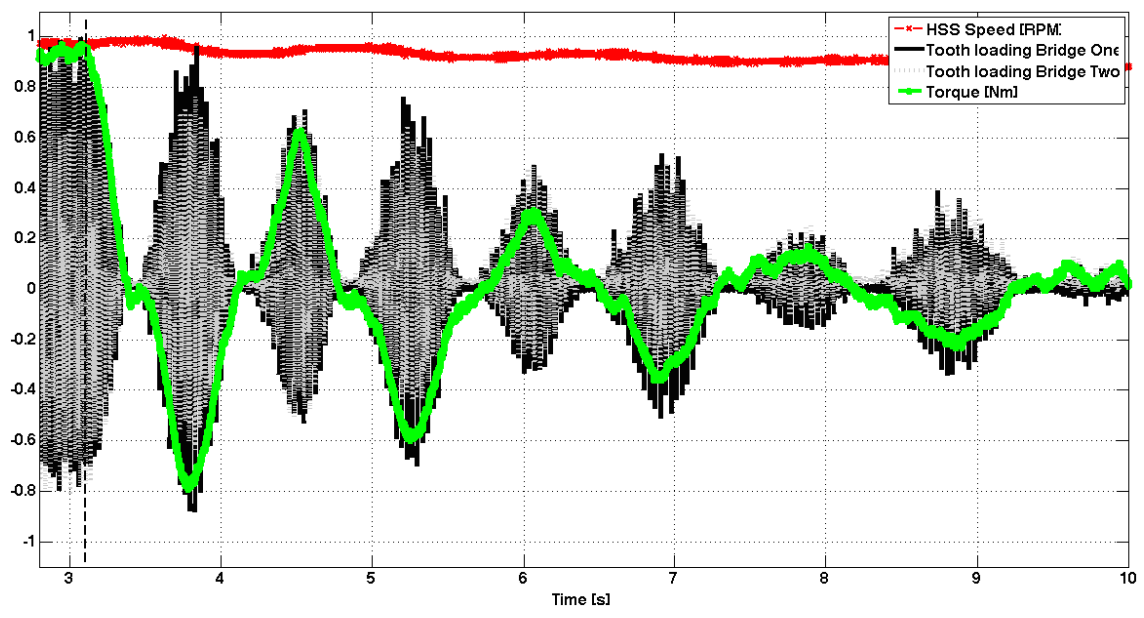

Figure 13: Tooth strains for gauge bridges 1 and 2 combined with normalized torque and normalized RPM signal for grid loss event.Gid loss start indicated with dashed line 


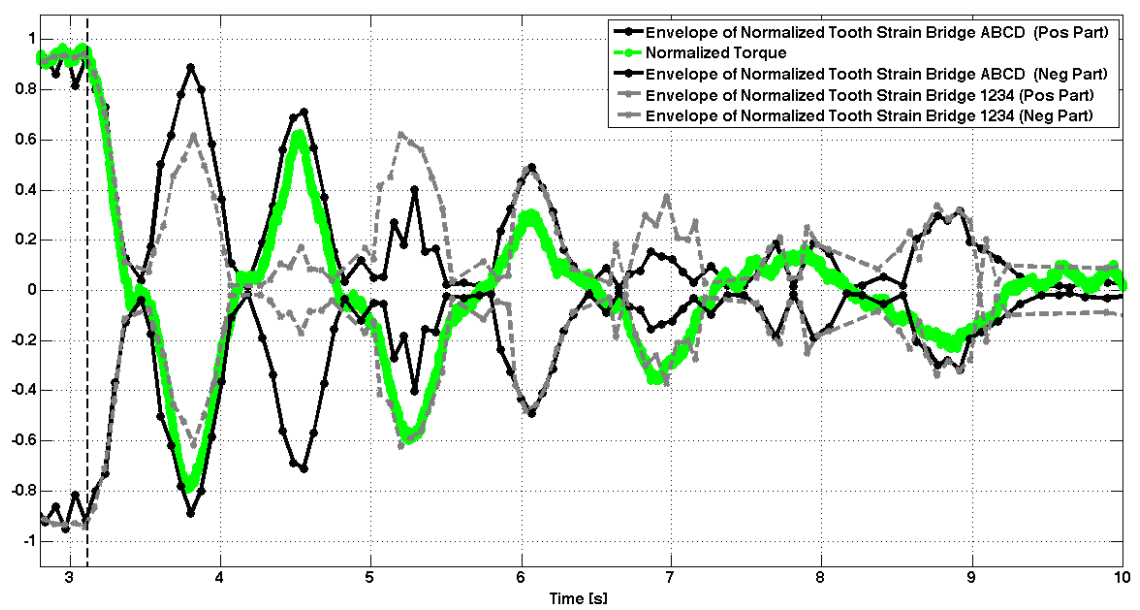

Figure 14: Envelopes of tooth strains for gauge bridges 1 and 2 combined with normalized torque signal for grid loss event. Gid loss start indicated with dashed line

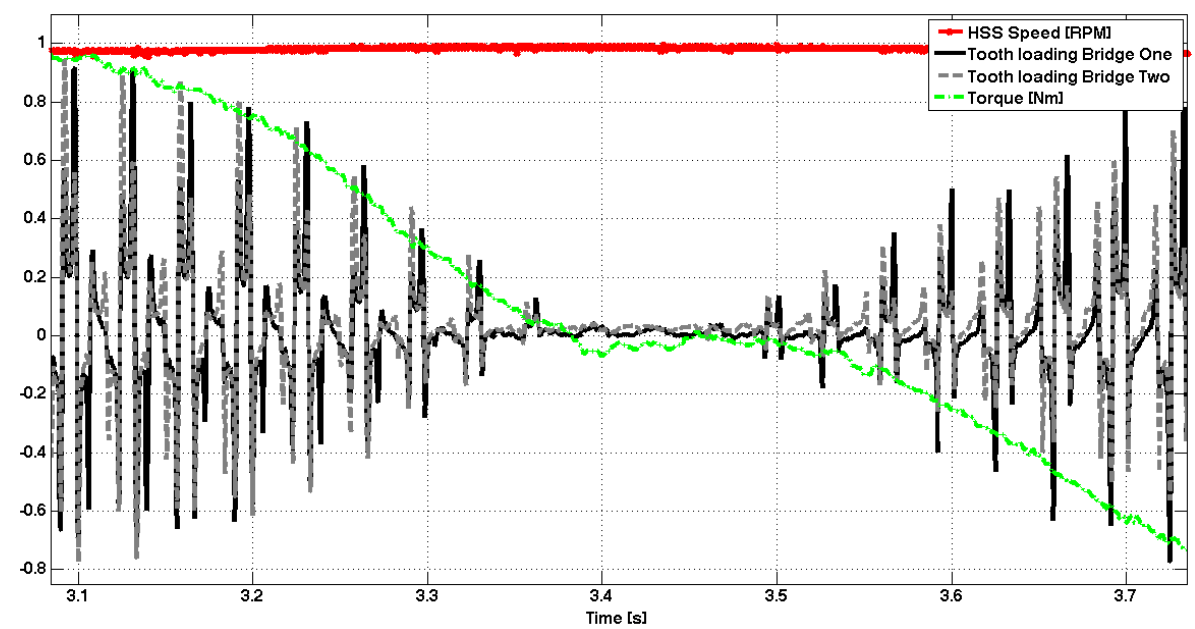

Figure 15: Tooth strains for gauge bridges 1 and 2 combined with normalized torque and normalized RPM signal for grid loss event. Zoom on torque reversal 


\section{Identification of driving eigenfrequency and modeshape}

\subsection{Polyreference least-squares complex frequency-domain}

The pLSCF estimation method is an extension of the LSCF method and was first introduced to find initial values for the iterative maximum likelihood method [16]. The LSCF method estimates a so-called common-denominator transfer function model [17]. Quickly it was found that the LSCF method yielded very clear stabilization diagrams with a very small computational effort $[18,19]$. In the pLSCF, the common-denominator transfer function model was replaced by a right matrix-fraction description (RMFD) transfer function model to obtain a truly poly-reference implementation [15]. The most important advantage of the LSCF estimator is the fact that very clear stabilization diagrams are obtained [20,21]. A complete background on frequency-domain system identification can be found in [22].

\subsection{Experimental identification}

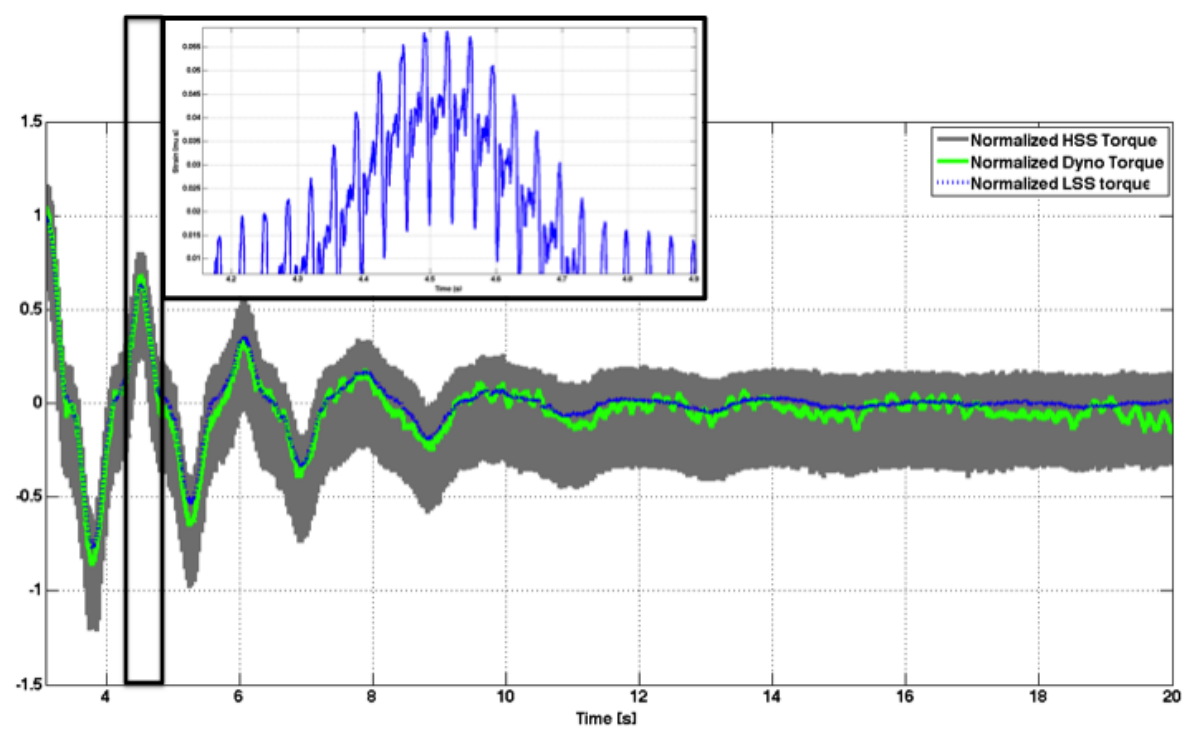

Figure 16: Signals Used in OMA analysis. Zoom on the HSS torque strain signal 


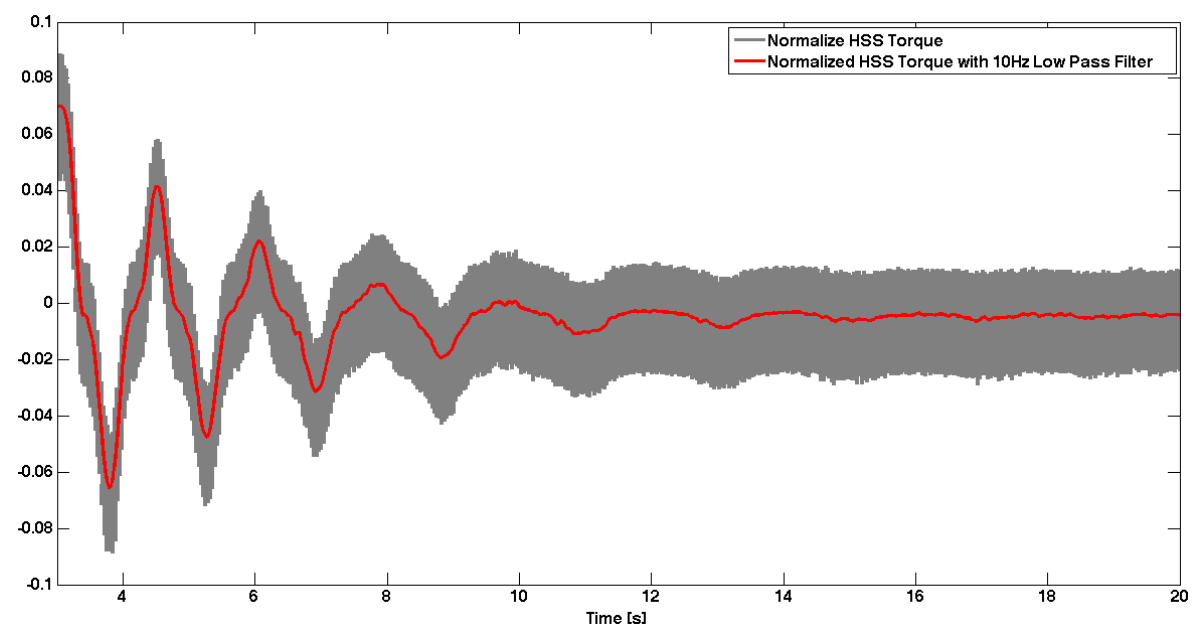

Figure 17: HSS torque strain signal filtered by means of low pass filter with $10 \mathrm{~Hz}$ filter frequency

Since the torque signal is clearly determining the overall behavior of the system it is expected that a torsional phenomenon is playing a crucial role in the event. The most likely driver of the behavior is the first drivetrain torsional resonance. The corresponding modeshape is characterized by counter phase rotation of the rotor and generator ineratia and is mainly determined by the rotor and generator inertia values and the drivetrain flexibility, illustrated in Figure 1. To perform modal identification an operational modal analysis is done on the torque strain gauge signals. Three signals are used: the torque signal measuring the dynamometer response, the LSS torque signal and the HSS torque signal. The discussed grid loss load case generates a broad excitation of the drivetrain and the decay of the signals is used in the operational modal analysis. Normalized signals used for visual illustration of the signal shape are shown in Figure 16. The high frequency fluctuation on the HSS torque signal in Figure 16 is shown in zoom on in the small subfigure of Figure 16. The fluctuation is related to a phenomenon above $10 \mathrm{~Hz}$, since it is absent in the $10 \mathrm{~Hz}$ low pass filtered HSS torque signal. Figure 17 illustrates this. Therefore, the high frequency behavior is outside the frequency range in which the first torsional drivetrain 
mode is expected.

An operational modal analysis (OMA) analysis is performed, using the decay of the signal to allow for accurate estimation of the damping value corresponding to the modeshape. Eigenfrequencies, modeshapes and damping estimates are generated directly by the pLSCF estimator. Figure 18 shows the FFTs of the used signals over a broad frequency range up to $80 \mathrm{~Hz}$. In this Figure a clear broadband excitation is present between 18 and $30 \mathrm{~Hz}$ and between 37 and $60 \mathrm{~Hz}$. These ranges correspond to a smearing of the nominal harmonic excitations at $30 \mathrm{~Hz}$ and $60 \mathrm{~Hz}$. Harmonic excitations are a challenge for the modal identification algorithms, since modal estimaters often misinterpret them as resonance frequencies. Figure 22 shows the pLSCF estimator stabilization diagram for a frequency band up to $50 \mathrm{~Hz}$. The y-axis shows the model order of the fitted modal model. One stable pole is present at $0.64 \mathrm{~Hz}$. No other stable poles were identified indicating that mainly a low frequency torsion based mode is excited. In the excitation ranges at $30 \mathrm{~Hz}$ and $60 \mathrm{~Hz}$ no modes were found by the pLSCF estimator. Therefore in this frequency range the modal identification is not disturbed by the harmonic excitation. 


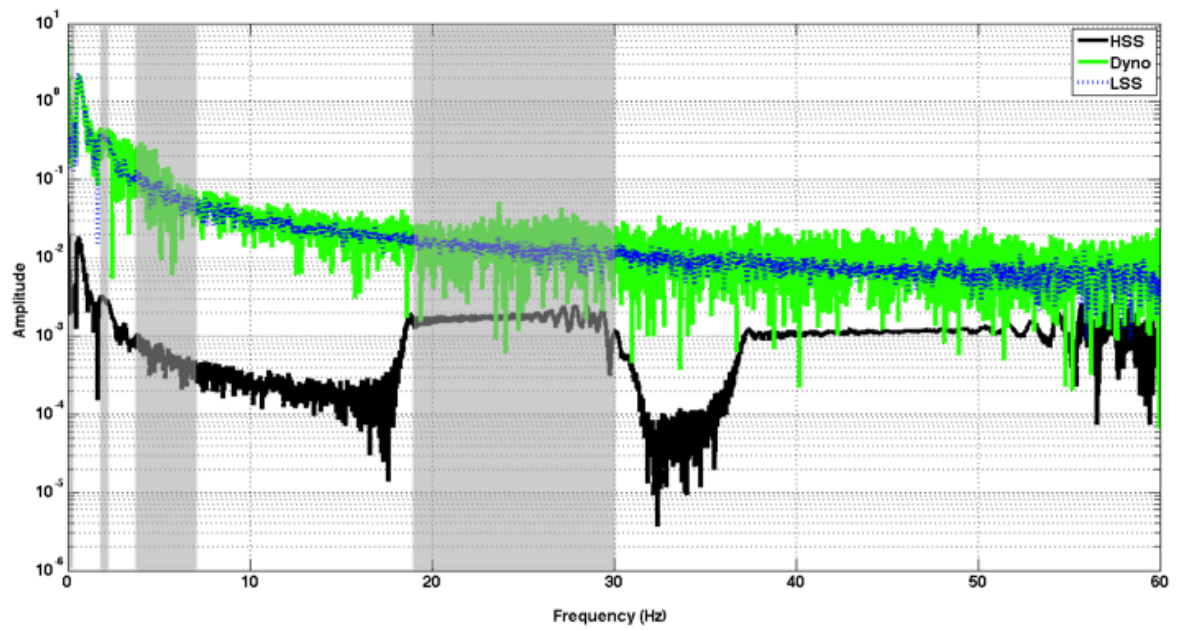

Figure 18: FFT spectra of Dyno Torque, LSS Torque and HSS Torque signals for frequency band $[0-80 \mathrm{~Hz}]$

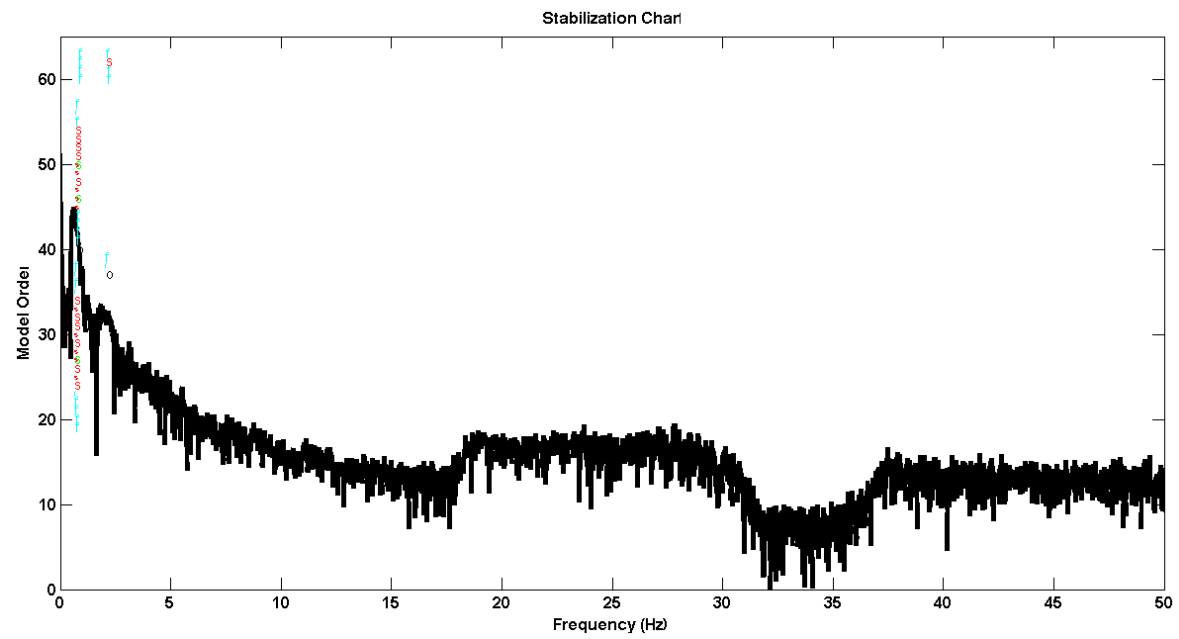

Figure 19: Stabilization diagram for pLSCF estimator eigenfrequency identification for frequency band $[0-50 \mathrm{~Hz}]$ 
To enhance the quality of the eigenfrequency estimation the modal identification focusses in this section on a smaller frequency range. Main goal is to identify whether the identified pole is a single pole as identified in Figure 22 or multiple poles are present in this frequency range. Figure 20 shows the FFTs of the signals in the zoomed frequency range. In grey the gear meshing excitation zones are shown. In these zones it is expected that the pLSCF could experience difficulties in performing the modal estimation. To assess estimation quality two model complexity levels are used. The latter will help to identify whether poles are expected to be physical or just mathematical noise of the modal fitting algorithm. The resulting stabilization diagrams for pole picking are shown in Figures 21 and 22 for a model order respectively of 32 and 64 .

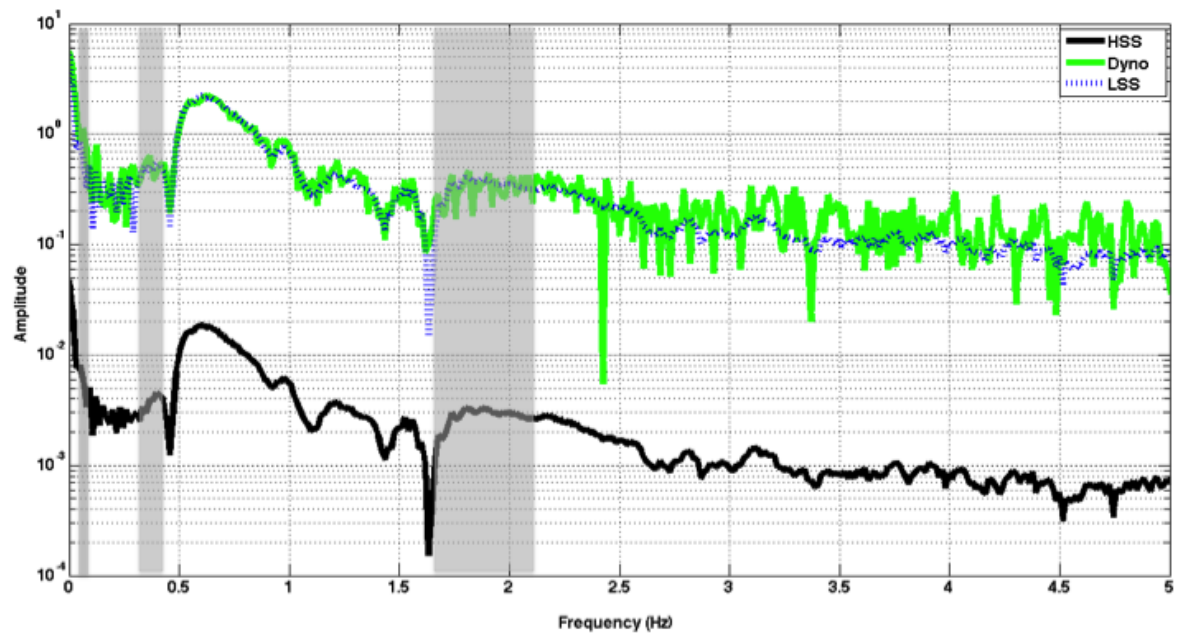

Figure 20: FFT spectra of Dyno Torque, LSS Torque and HSS Torque signals 


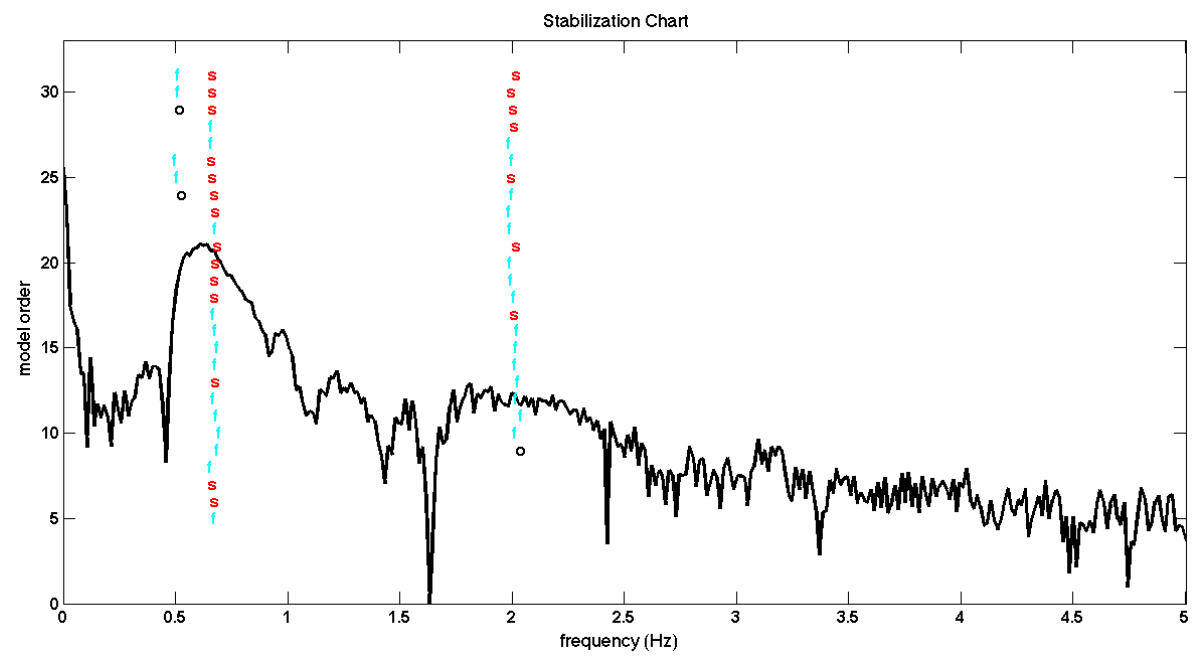

Figure 21: Stabilization diagram for Polymax eigenfrequency identification for frequency band [0-5Hz] for model order 32

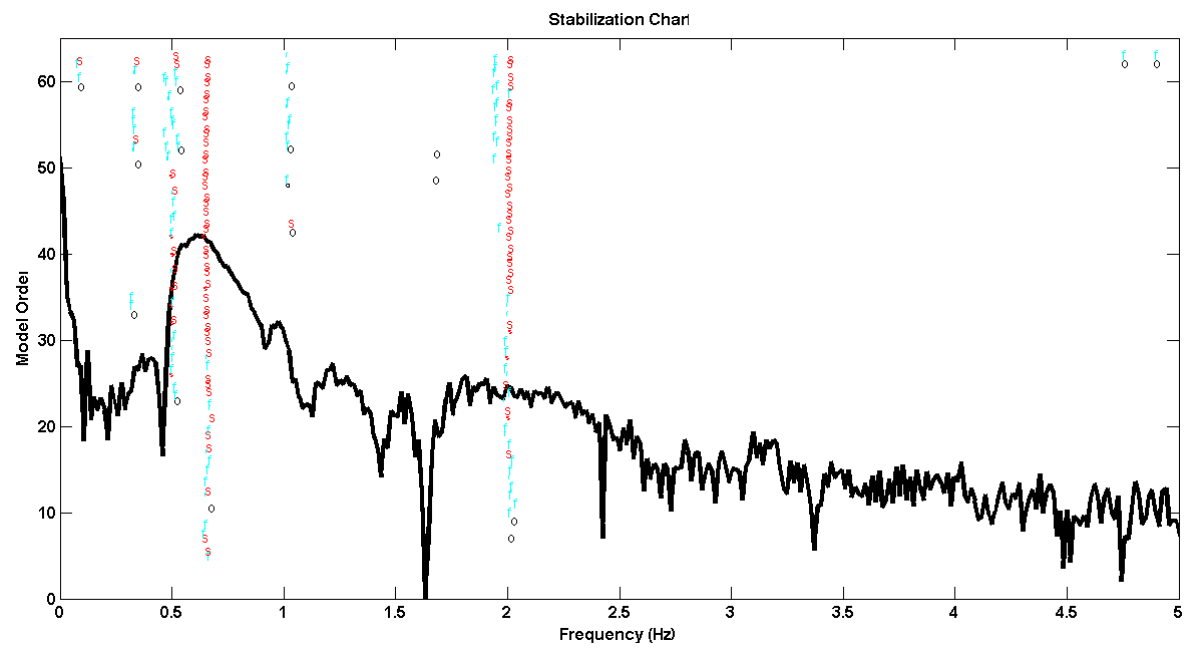

Figure 22: Stabilization diagram for pLSCF estimator eigenfrequency identification for frequency band $[0-5 \mathrm{~Hz}]$ for model order 64 
Three poles are found/ Two poles, respectively at $0.64 \mathrm{~Hz}$ and at $1.99 \mathrm{~Hz}$ are stable for multiple model complexity levels in both analyses. The first pole at $0.49 \mathrm{~Hz}$ shows some stable estimations for only a limited number of model orders. Figure 23 shows the eigenfrequencies for the poles estimated as stable for the different model orders in the stabilization diagram shown in Figure 22 . Stable values are found for all three frequencies. Nonetheless only two eigenfrequencies have a significant amount of estimations. Corresponding damping values are shown in Figure 24. Two identified modes have low damping values whereas one modeshape has a higher damping value, which could be related to the fact that the speed is brought to zero in a controlled manner by the dyno controller thereby counteracting the system response. The main characteristic of the torsional modes is the phase behaviour of the rotor inertia and generator rotor inertia. Figure 25 indicates the phase difference between the modeshape rotational displacements of those inertias. All modes show a phase difference of about $\pi$ radians, corresponding to the behaviour shown in Figure 1.

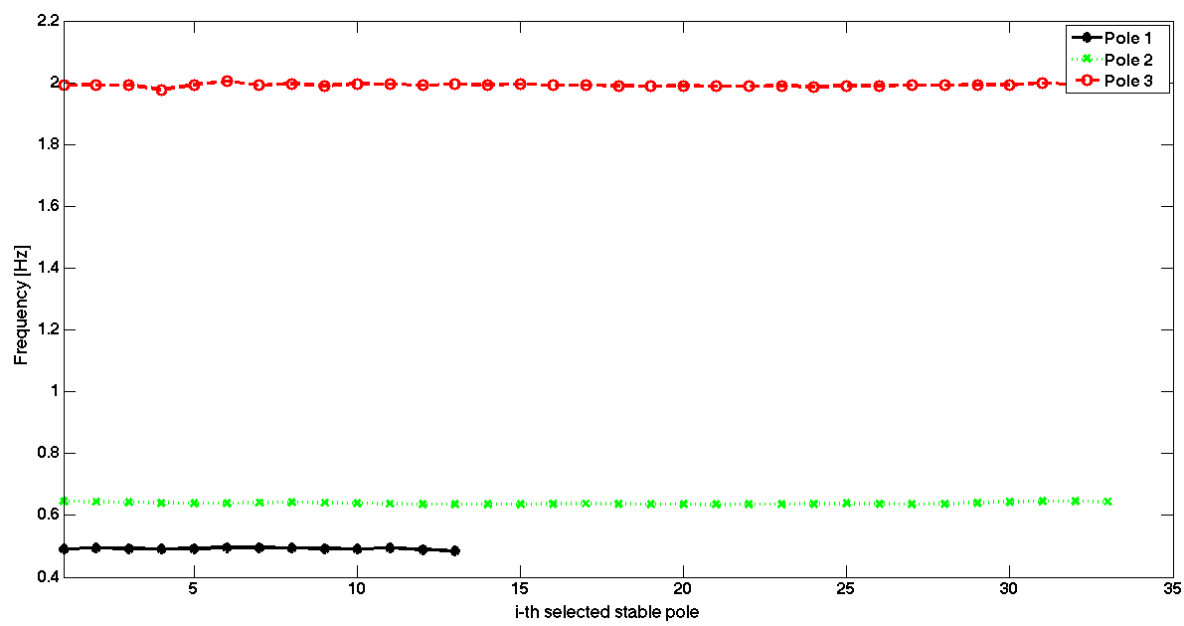

Figure 23: Estimated eigenfrequencies for selected stable poles in stabilization diagram shown in Figure 22 


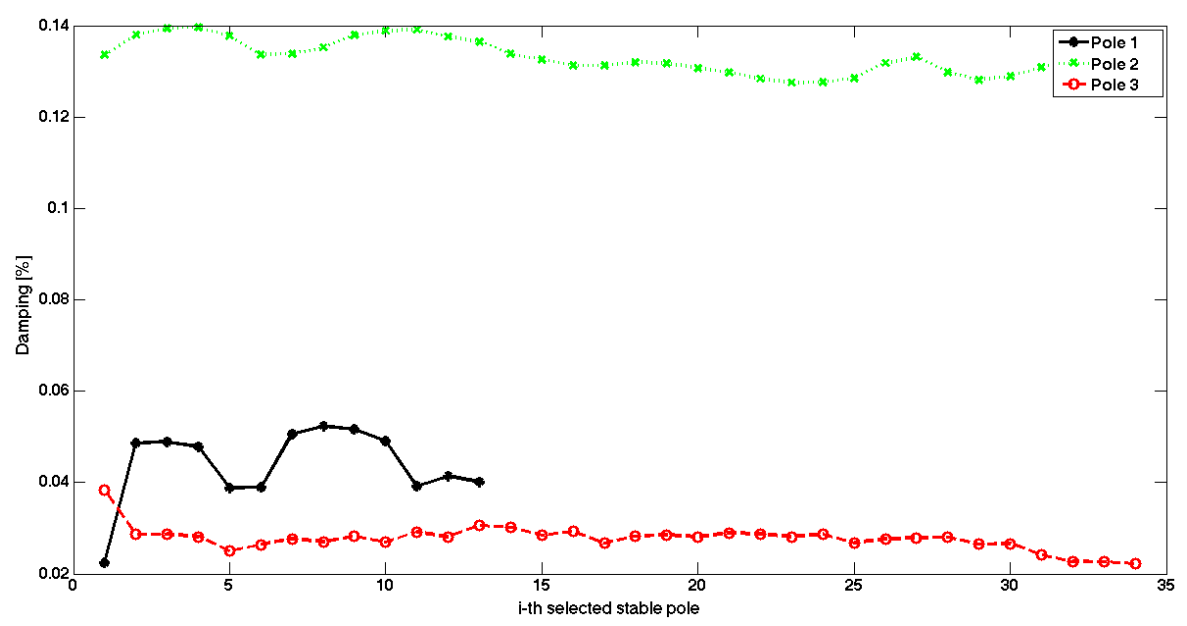

Figure 24: Damping values for selected stable poles in stabilization diagram shown in Figure 22

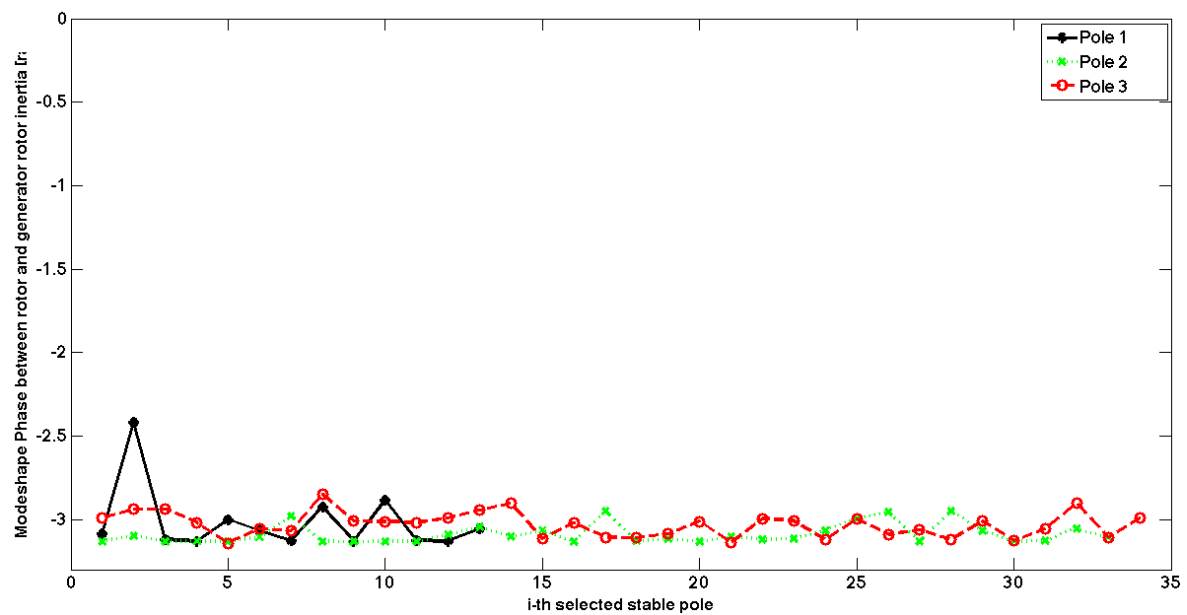

Figure 25: Phase difference between rotor and generator inertia for selected stable poles in stabilization diagram shown in Figure 22 
Since significant gear related harmonic excitation is present in the system the OMA resonance estimates should be handled with care. In the OMA analysis no input forces are measured. Therefore attention should be paid to identify and separate the harmonic components from the structural modes and to reduce their influence on the extracted modal parameters. Jacobsen [23] showed that there can be significant bias on the modal parameters obtained from OMA or even no estimated structural modes. Therefore potentially not all identified resonances are physical but also mathematical modes can result from the estimator. To get insights in the quality of the estimated modes a synthesis of the frequency response functions (FRFs) is performed.
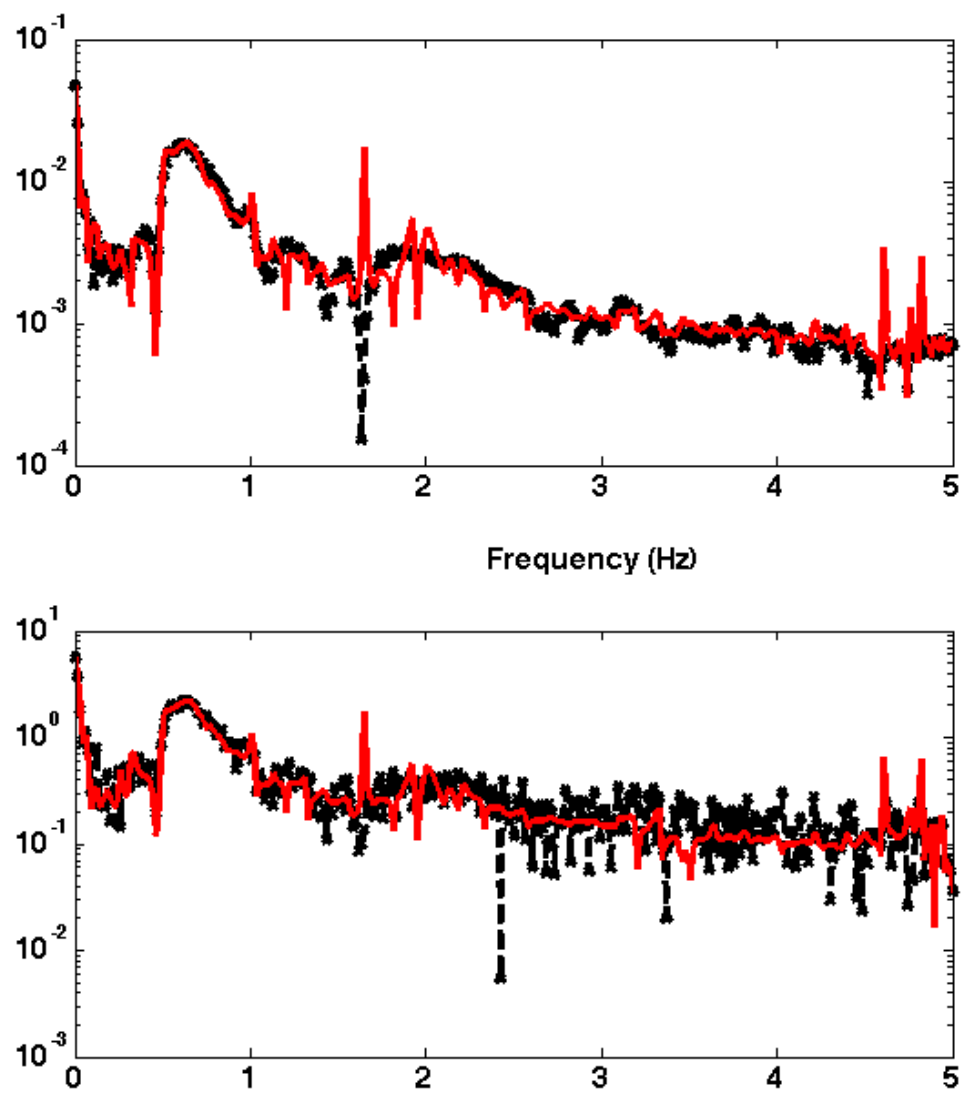

Figure 26: Modal synthesis results using estimated eigenfrequencies and modeshapes. Black dashed curve: Measurements Red curve: Synthesis 
In such an FRF synthesis a limited number of eigenfrequencies and modeshapes is used to describe the system response. For proportionally damped systems this relationship between the synthesised FRF matrix $H\left(\omega_{k}\right)$ and the modeshapes is expressed by:

$$
H\left(\omega_{k}\right)=\sum_{i=1}^{N} \frac{\{\phi\}_{i}\{\phi\}_{i}^{T}}{\left(\omega_{n_{i}}^{2}-\omega_{k}^{2}\right)+j \cdot 2 \cdot \xi_{i} \omega_{n_{i}} \omega_{k}}
$$

with:

$\mathrm{N}$ the number of calculated modes

$\{\phi\}_{i}$ the i-th mass normalized modeshape

$\omega_{n_{i}}$ the i-th natural frequency

$\xi_{i}$ the i-th modal damping ratio

Results are shown in Figure 26. In the zones dominated by harmonic excitation around $0.4 \mathrm{~Hz}$ and $2 \mathrm{~Hz}$, shown in grey in Figure 20 the FRF synthesis is of low quality. Peaks are present, which were not found in the measured signal potentially indicating the presence of mathematical poles.

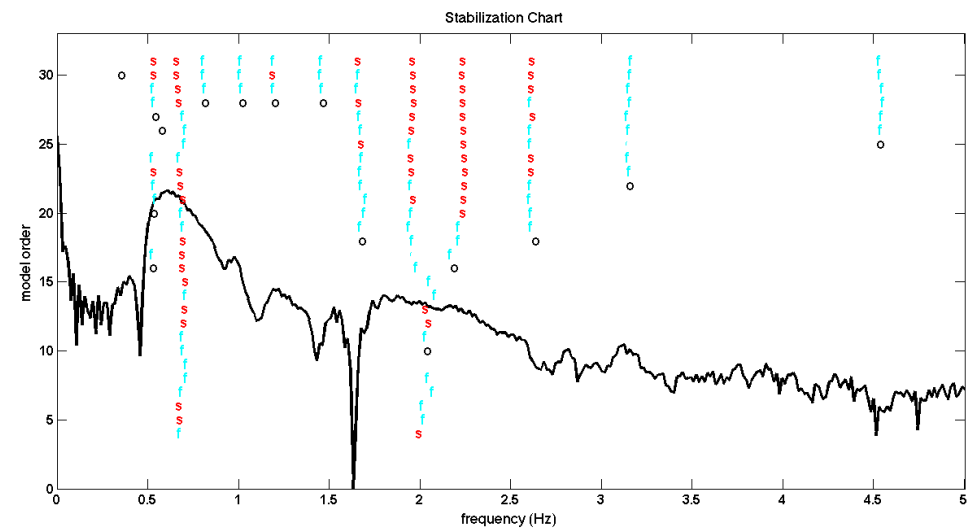

Figure 27: Stabilization diagram for pLSCF estimator eigenfrequency identification for frequency band $[0-5 \mathrm{~Hz}]$ 
Based on the synthesis results and the low number of stable model orders for the pole at $0.49 \mathrm{~Hz}$ in the stabilization diagrams it is concluded that this pole is not a physical resonance. The same is expected for the pole estimated at $2 \mathrm{~Hz}$. To strengthen this conclusion Figure 27 shows the stabilization diagram when only the LSS Torque and HSS Torque signals are taken into account in the OMA estimation. In this case the pole is unstable for lower model order and splits in two poles for higher model orders. The latter in combination with the clear presence of harmonic excitation and non-stable FRF synthesis results indicates that the estimated pole is not a resonance frequency but a harmonic excitation. Therefore it can be concluded that the grid loss event driving torsional modeshape has a corresponding eigenfrequency at $0.64 \mathrm{~Hz}$.

\section{Conclusions}

This paper experimentally investigated a grid loss event on the NREL nacelle testrig. The system vibration was driven by a modeshape characterized by a counter phase rotation of the rotor and generator rotor about the drivetrain flexibility. This behavior results in significant torque oscillations with significant negative torque periods. At each zero-crossing the teeth disengage contact, go through the backlash and re-engage at the other flank. Strain gauge measurements at the HSS pinion teeth were used to show this. The driving torsional resonance was identified by means of an pLSCF estimator resulting in eigenfrequency, damping and modeshape. Harmonic excitation originating from the gears had a clear influence on the estimation and resulted in mathematical modes. It was shown that these modes were non-physical. However these harmonic excitations modes were not present in the frequency range of the torsional mode of interest resulting in a clear estimation of the eigenfrequency at $0.64 \mathrm{~Hz}$ and its corresponding damping value and modeshape. The knowledge of this eigenfrequency and modeshape gives insights in the dominant response of the system during grid loss without external braking. 


\section{Aknowledgements}

This research was performed in the framework of different support schemes of the Instituut voor Wetenschap en Technologie IWT Vlaanderen and Fonds Wetenschappelijk Onderzoek (FWO) Vlaanderen

The National Renewable Energy Laboratory's GRC is funded by the Wind and Water Power Program, Office of Energy Efficiency and Renewable Energy of the U.S. Department of Energy (No DE-AC36-08GO28308) under Contract No. DE-AC02-05CH11231. The U.S. Department of Energy is recognized for its continued support of the GRC project.

\section{Bibliography}

[1] H.Long, I.S.Al-Tubi: Effects of operational conditions on wind turbine gear micropitting, Proceedings of European Wind Energy Association Conference EWEA, Vienna, 2013

[2] H.Link, J.Keller, Y.Guo, B.McNiff: Gearbox Reliability Collaborative: Phase 3 gearbox 2 test plan, NREL Technical Report NREL/TP-500058190,2013

[3] J.Helsen, P.Peeters, K.Vanslambrouck, F.Vanhollebeke, W.Desmet: The dynamic behavior induced by different wind turbine gearbox suspension methods assessed by means of the flexible multibody technique, Renewable Energy, Vol. 69, pp. 336-346, 2014

[4] J.Mann: Wind field simulation, Prob Eng Mech, Vol.13 (4), pp. 269282, 1998

[5] J. Peeters: Simulation of dynamic drive train loads in a wind turbine, PhD thesis Katholieke Universiteit Leuven, Department of Mechanical Engineering, Division PMA, Leuven (Heverlee), Belgium (2006) Available online: http://hdl.handle.net/1979/344 
[6] Y.Guo, J.Keller, W.LaCava: Combined effects of gravity, bending moment, bearings clearance and input torque on wind turbine planetary gear load sharing, Proceedings of AGMA Fall Technical Meeting, Dearborn Michigan, 2012

[7] W.LaCava, Y.Xing, C.Marks, Y.Guo, T.Moan: 'Three-dimensional bearing load share behaviour in the planetary stage of a wind turbine gearbox', IET Renewable Power Generation, vol. 7(4), p. 359-369, 2013

[8] L.Hui, M.Steurer, K.L.Shi, S.Woodruff, Da Zhang: Development of a Unified Design, Test, and Research Platform for Wind Energy Systems Based on Hardware-in-the-Loop Real-Time Simulation, Industrial Electronics, IEEE Transactions on , vol.53(4), pp.1144,1151, 2006

[9] J.Helsen, B.Marrant, F.Vanhollebeke, F.De Coninck, D.Berckmans, D.Vandepitte, W.Desmet: Assessment of excitation mechanisms and structural flexibility influence in excitation propagation in multi-megawatt wind turbine gearboxes: Experiments and flexible multibody model optimization, Mechanical Systems and Signal Processing, Vol. 40(1),pp. 114-135, 2013

[10] S.Struggl, V.Berbyuk, H.Johansson: Review on wind turbines with focus on drive train system dynamics, Wind Energy, 2014, DOI: 10.1002/we.1721

[11] International electrotechnical commission (IEC) Standard. Wind turbines - part 1: Design requirements, 2005.

[12] International electrotechnical commission (IEC) Standard. Wind turbines - Part 4: design requirements for WT gearboxes, 2005.

[13] J. Peeters, S. Goris, F. Vanhollebeke, B. Marrant, W. Meeussen, J. Helsen, D. Vandepitte, W. Desmet: Insights in multimegawatt wind turbine gearbox dynamics using a validated multibody modelling appraoch, Proceedings of ATK (Aken, Germany), 2009 
[14] H.Link, W.LaCava, J.Van Dam, B.McNiff, S.Sheng, R.Wallen, M.McDade, S.Lambert, S.Butterfield, F.Oyague: Gearbox Reliability Collaborative Project Report: Findings From Phase 1 and Phase 2 Testing, NREL Technical Report: NREL/TP-5000-51885, Available online at http://www.nrel.gov/docs/fy11osti/51885.pdf

[15] P. Guillaume, P. Verboven, S. Vanlanduit, H. Van Der Auweraer, B. Peeters: A poly-reference implementation of the least-squares complex frequency-domain estimator, Proceedings of IMAC vol. 21, p.183-192, 2003

[16] P. Guillaume, P. Verboven, S. Vanlanduit: Frequency-domain maximum likelihood identification of modal parameters with confidence intervals, Proceedings of ISMA 23, the International Conference on Noise and Vibration Engineering, Leuven, Belgium, 1998

[17] P. Guillaume, R. Pintelon, J. Schoukens: Parametric identification of multivariable systems in the frequency domain - a survey, Proceedings of ISMA 21, the International Conference on Noise and Vibration Engineering, Leuven, Belgium, 1996

[18] H. Van Der Auweraer, P. Guillaume, P. Verboven, S. Vanlanduit: Application of a fast stabilizing frequency domain parameter estimation method. ASME Journal of Dynamic Systems, Measurement, and Control, vol.123(4), pp.651658, 2001

[19] P. Verboven: Frequency domain system identification for modal analysis, PhD Thesis, Vrije Universiteit Brussel, Belgium, 2002

[20] B. Peeters, P. Guillaume, H. Van Der Auweraer, B. Cauberghe, P. Verboven, J. Leuridan: Automotive and aerospace applications of the LMS PolyMAX modal parameter estimation method, Proceedings of IMAC 22, Dearborn (MI), USA, 2004

[21] B. Peeters, H. Van Der Auweraer, P. Guillaume, J. Leuridan: The Poly- 
MAX frequency-domain method: a new standard for modal parameter estimation, Shock and Vibration, vol.11(3-4), pp. 395-409, 2004.

[22] R. Pintelon, J. Schoukens: System Identification: a Frequency Domain Approach. IEEE Press, New York, 2001

[23] N-J. Jacobsen: Separating Structural Modes and Harmonic Components in Operational Modal Analysis, Proceedings of the International Modal Analysis Conference (IMAC) XXIV, St. Louis, USA, 2006 